\title{
V1309 Scorpii: merger of a contact binary ${ }^{\star, \star \star}$
}

\author{
R. Tylenda ${ }^{1}$, M. Hajduk ${ }^{1}$, T. Kamiński ${ }^{1}$, A. Udalski ${ }^{2,3}$, I. Soszyński ${ }^{2,3}$, M. K. Szymański²,3, \\ M. Kubiak ${ }^{2,3}$, G. Pietrzyński ${ }^{2,3,4}$, R. Poleski ${ }^{2,3}$, Ł. Wyrzykowski ${ }^{3,5}$, and K. Ulaczyk ${ }^{2,3}$ \\ ${ }^{1}$ Department for Astrophysics, N. Copernicus Astronomical Center, Rabiańska 8, 87-100 Toruń, Poland \\ e-mail: [tylenda; cinek; tomkam] ancac. torun.pl \\ 2 Warsaw University Observatory, Al. Ujazdowskie 4, 00-478 Warsaw, Poland \\ e-mail: [udalski; soszynsk;msz;mk; pietrzyn;rpoleski; kulaczyk]@astrouw.edu.pl \\ 3 The Optical Gravitational Lensing Experiment, Poland \\ ${ }^{4}$ Universidad de Concepción, Departamento de Astronomia, Casilla 160-C, Concepción, Chile \\ 5 Institute of Astronomy, University of Cambridge, Madingley Road, Cambridge CB3 OHA, UK \\ e-mail: wyrzykow@ast.cam.ac.uk
}

Received 29 November 2010 / Accepted 1 February 2011

\begin{abstract}
Context. Stellar mergers are expected to take place in numerous circumstences in the evolution of stellar systems. In particular, they are considered as a plausible origin of stellar eruptions of the V838 Mon type. V1309 Sco is the most recent eruption of this type in our Galaxy. The object was discovered in September 2008.

Aims. Our aim is to investigate the nature of V1309 Sco.

Methods. V1309 Sco has been photometrically observed in course of the OGLE project since August 2001. We analyse these observations in different ways. In particular, periodogram analyses were done to investigate the nature of the observed short-term variability of the progenitor.

Results. We find that the progenitor of V1309 Sco was a contact binary with an orbital period of $\sim 1.4$ day. This period was decreasing with time. The light curve of the binary was also evolving, indicating that the system evolved towards its merger. The violent phase of the merger, marked by the systematic brightenning of the object, began in March 2008, i.e. half a year before the outburst discovery. We also investigate the observations of V1309 Sco during the outburst and the decline and show that they can be fully accounted for within the merger hypothesis.

Conclusions. For the first time in the literature we show from direct observations that contact binaries indeed end up by merging into a single object, as was suggested in numerous theoretical studies of these systems. Our study also shows that stellar mergers indeed result in eruptions of the V838 Mon type.
\end{abstract}

Key words. stars: individual: V1309 Sco - binaries: general - stars: peculiar

\section{Introduction}

Stellar mergers have for a long time been recognized to play an important role in the evolution of stellar systems. High stellar densities in globular clusters can lead often to collisions and mergers of stars (Leonard 1989). In this way the origin of blue strugglers can be explained. In dense cores of young clusters multiple mergers of protostars have been suggested as a way of formation of the most massive stars (Bonnell et al. 1998). Some binary stars, in particular contact binaries, are suggested to end their evolution as stellar mergers (Robertson \& Eggleton 1977).

The powerful outburst of V838 Mon in 2002 (Munari et al. 2002), accompanied by a spectacular light echo (Bond et al. 2003), raised interest in a class of stellar eruptions named "red novae", "optical transients" or "V838 Mon type eruptions". These objects, which typically reach a maximum luminosity of $\sim 10^{6} L_{\odot}$, evolve to low effective temperatures and decline as

\footnotetext{
* Based on observations obtained with the 1.3-m Warsaw telescope at the Las Campanas Observatory of the Carnegie Institution of Washington.

$\star \star$ The photometric data analysed in the present paper are available from the OGLE Internet archive:

ftp://ogle.astrouw.edu.pl/ogle/ogle3/V1309_sCO
}

very cool (super)giants. Apart form V838 Mon, in our Galaxy the class also includes V4332 Sgr, whose outburst was observed in 1994 (Martini et al. 1999), and V1309 Sco, which erupted in 2008 (Mason et al. 2010). As extragalactic eruptions of this kind one can mention M 31 RV (eruption in 1989, Mould et al. 1990), M 85 OT2006 (Kulkarni et al. 2007), and NGC300 OT2008 (Berger et al. 2009). Several interpretations of the eruptions were proposed. They include an unusual classical nova (Iben \& Tutukov 1992; Shara et al. 2010), a late He-shell flash (Lawlor 2005), or a thermonuclear shell flash in an evolved massive star (Munari et al. 2005). Tylenda \& Soker (2006) presented numerous arguments against these mechanisms. They showed that all the main observational characteristics of the V838 Montype eruptions can be consistently understood as resulting from stellar collisions and mergers, as originally proposed in Soker \& Tylenda (2003). For these reasons Soker \& Tylenda (2007) proposed to call these type of eruptions mergebursts. Recently, Kashi et al. (2010) and Kashi \& Soker (2011) have suggested that some of the V838 Mon-type eruptions are of the same nature as the eruptions of luminous blue variables, and that they can be powered by mass-transfer events in binary systems.

In the present paper, we show that the recent red nova, V1309 Sco, is a Rosetta stone in the studies of the nature of 
the V838 Mon type eruptions. Archive photometric data collected for the object in the OGLE project during about six years before the outburst allow us to conclude that the progenitor of V1309 Sco was a contact binary. The system quickly evolved towards its merger, which resulted in the eruption observed in 2008.

\section{V1309 Scorpii}

V1309 Sco, also known as Nova Sco 2008, was discovered on 2.5 September 2008 (JD 2454712 ) (Nakano 2008). The subsequent evolution, however, showed that as pointed out in Mason et al. (2010), this was not a typical classical nova.

Early spectroscopy revealed an F-type giant (Rudy et al. 2008a). On a time scale of a month the object evolved to Kand early M-types (Mason et al. 2010; Rudy et al. 2008b). Eight months after the discovery it was observed as a late M-type giant (Mason et al. 2010).

As described in Mason et al. (2010), the object developed a complex and rapidly evolving line spectrum of neutral and singly ionized elements. In early epochs, absorption features dominated the spectrum, emission components developed later and their intensities quickly increased with time. The strongest emission lines were those of the hydrogen Balmer series.

The emission components had FWHM of $\sim 150 \mathrm{~km} \mathrm{~s}^{-1}$ and broader wings, which in the case of $\mathrm{H} \alpha$ extended even beyond $1000 \mathrm{~km} \mathrm{~s}^{-1}$ (Mason et al. 2010). Narrow absorption components were superimposed on the emission components, so that the line profiles mimicked those of P-Cyg type or inverse P-Cyg in some cases. Mason et al. (2010) interpret these line profiles as produced in an expanding shell that is denser in the equatorial plane.

In late epochs, when the object evolved to M-type, absorption features of $\mathrm{TiO}, \mathrm{VO}, \mathrm{CO}$, and $\mathrm{H}_{2} \mathrm{O}$ appeared in the spectrum (Mason et al. 2010; Rudy et al. 2008b).

V1309 Sco thus shares the principal characteristic of the V838 Mon type eruptions, i.e evolution to very low effective tempratures after maximum brightness and during the decline (Tylenda \& Soker 2006). Other common features of V1309 Sco and the V838 Mon type eruptions include: outburst time scale of the order of months, outburst amplitude (maximum minus progenitor) of 7-10 mag, complete lack of any high-ionization features (coronal lines, in particular), expansion velocities of a few hundred $\mathrm{km} \mathrm{s}^{-1}$ (instead of a few thousands as in classical novae), and oxide bands observed in later epochs, which imply that oxygen-rich $(\mathrm{C} / \mathrm{O}<1)$ matter was involved in the eruption.

\section{Observations}

Owing to the position of V1309 Sco close to the Galactic centre $\left(l=359^{\circ} .8, b=-3^{\circ} .1\right)$, the object appears to be situated within a field monitored in the OGLE-III and OGLE-IV projects (Udalski $2003)^{1}$. As a result V1309 Sco was observed on numerous occasions since August 2001. Altogether more than 2000 measurements were obtained predominantly in the I Cousins photometric band. Among them, 1340 observations were made before the discovery of the object as a nova in September 2008. A few observations were also made in the $V$ band (seven before the discovery). The data were reduced and calibrated using standard OGLE procedures (Udalski et al. 2008). A typical precision of the measurements was $0.01 \mathrm{mag}$.

\footnotetext{
${ }^{1}$ http://ogle.astrouw.edu.pl
}

\section{Results}

The entire measurements of V1309 Sco derived from the OGLEIII and IV surveys in the $I$ photometric band are displayed in Fig. 1. The gaps in the data are owing to conjunctions of the object with the Sun. Apart from 2001 and 2009 most of the data were obtained between February and October of each year. In 2001 the OGLE-III project was just starting to operate, hence only 11 measurements were obtained in this year (they were omitted from the analysis discribed below). In the period 2002-2008, from 52 (2002, 2003) to 367 (2006) observations were made each year. Near the maximum of the 2008 eruption, i.e. when the object was brighter than $I \simeq 11$, its image was overexposed in the OGLE frames, hence there are no data for this period. Near maximum brightness, the object attained $I \simeq 6.8$, according to the data gathered in $\mathrm{AAVSO}^{2}$. In May 2009 OGLE-III phase ended and threfore only 64 data points were collected during the 2009 observing season. The OGLE project resumed regular observations of the Galactic centre in March 2010 with a new instrumental setup, namely a 32 chip mosaic camera, which started the OGLE-IV phase. In 2010655 measurements were obtained.

As can be seen from Fig. 1, the progenitor of V1309 Sco was initially slowly increasing in brightness on a time scale of years and reached a local maximum in April 2007. Subsequently, the star faded by $\sim 1$ mag during a year. In March 2008, the main eruption started, which led to the object discovery, as Nova Sco 2008 , half a year later. Remarkable is the smooth, roughly exponential rise in brightness. During this event the object brightened by $\sim 10 \mathrm{mag}$, i.e. by a factor of $10^{4}$. An analysis of the data for the progenitor (2002-2007) is presented in Sect. 5, while the outburst and the decline of the object are discussed in Sect. 6.

\section{The progenitor}

\subsection{The data}

A short-term variability, resulting in a $\sim 0.5 \mathrm{mag}$ scatter of the points in Fig. 1, is the most remarkable and interesting feature of the V1309 Sco progenitor. This variability is strictly periodic. To show this, we used a method that employs periodic orthogonal polynomials to fit the observations and an analysis of variance to evaluate the quality of the fit, as described in SchwarzenbergCzerny (1996). This method is particularly suitable for analysing unevenly sampled data of non-sinusoidal periodic variations, such as eclipses in binary systems. The resulting periodograms obtained for the particular observational seasons are presented in Appendix A. They show that the principal periodicity in the variations observed in seasons 2002-2007 corresponds to a period of $\sim 1.4$ day. However, the derived period was not constant, but slowly decreasing with time, as presented in Fig. 2. It decreased by $1.2 \%$ during the pre-outburst observations.

When deriving the period plotted in Fig. 2, we devided the data sequence available for a given season into subsamples, each containing $\sim 50$ data points, and constructed periodograms for each subsample separately. In this way we can see that during later seasons, particularly in 2006 and 2007, the period was significantly varying on a time scale of months.

We find that the time evolution of the observed period can be fitted by an exponential formula. A result of a least-squares

2 Available at http://www . aavso.org/ 


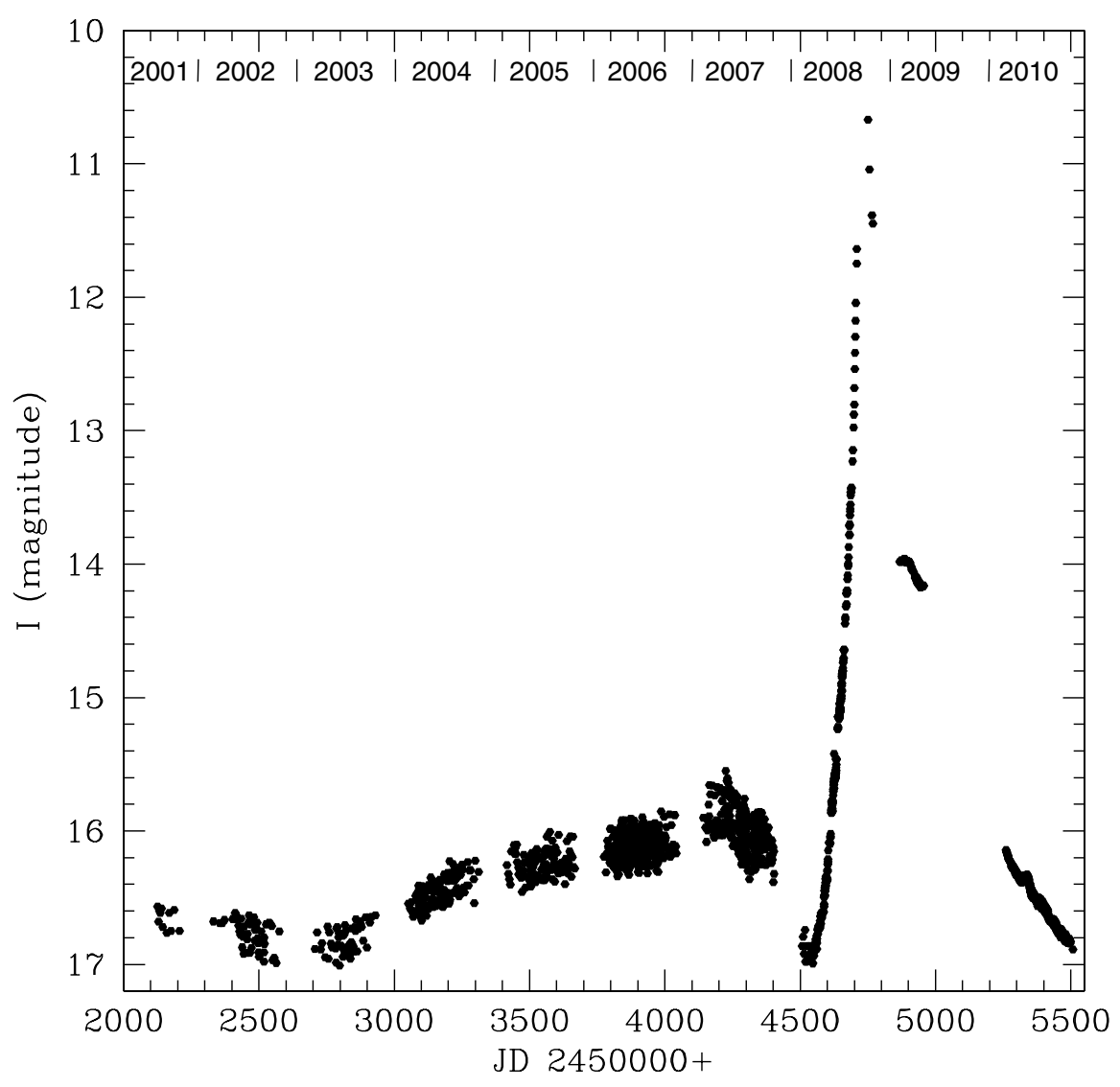

Fig. 1. Light curve of V1309 Sco from the OGLE-III and OGLE-IV projects: I magnitude versus time of observations in Julian Dates. Time in years is marked on top of the figure. At maximum the object attained $I \simeq 6.8$. fit (inverse errors used as weights) is shown with the full line in Fig. 2. The obtained formula is

$P=1.4456 \exp \left(\frac{15.29}{t-t_{0}}\right)$

where $P$ is the period in days, $t$ is the time of observations in Julian Dates, and $t_{0}=2455$ 233.5.

Using the above period fit we folded the observations and obtained light curves of the object in particular seasons. The results are displayed in Fig. 3. In 2002-2006 the light curves were obtained from all available data for a particular seasons. The results are shown in the upper part of Fig. 3. In 2007 the light curve significantly evolved during the season and therefore we plotted the results for the subsamples (the same as when deriving the period) of 2007 season in the lower part of Fig. 3 (time goes from subsample a to e in the figure).

As can be seen from Fig. 3, the light curve in 2002-2006 displays two maxima and two minima. They are practically equally spaced in the phase and have similar shapes in the early seasons. This is the reason why the periodograms for these seasons (see Appendix A) show a strong peak at a frequency twice as high (a period twice as short) as the main peak. In other words, the observations could have been interpreted with a period of $\sim 0.7$ day and a light curve with one maximum and minimum. The periodograms show however already in the 2002 season that the period that is twice as long ( $\sim 1.4$ day) better reproduces the observations. The reason is obvious in the later seasons, when the first maximum (at phase 0.25 in Fig. 3) in the light curve becomes increasingly stronger than the second one. As a result the peak corresponding to the 0.7 day period deacreases and practically disappears in 2007.

\subsection{Interpretation}

In principle one can consider three possible interpretations of the observed light variability, i.e. stellar pulsation, single-star rotation, and an eclipsing binary system.

\subsubsection{Pulsation}

The light curve in the early seasons may imply a stellar pulsation with a period of $\sim 0.7$ day and an amplitude of $\sim 0.15$ mag. This interpretation, however, encounters severe problems when it is used to explain the observed evolution of V1309 Sco. As we show below, the progenitor was probably a K-type star. There is no class of pulsating stars that could be reconciled with these characteristics, i.e. a K-type star pulsating with the above period and amplitude. Moreover, to explain the observed evolution of the light curve displayed in Fig. 3, one would have to postulate a switch of the star pulsation to a period exactly twice as long on a time scale of a few years, together with a gradual shortening of the period. This would be very difficult, if not impossible, to understand within the present theory of stellar pulsation. Finally, there is no physical mechanism involving or resulting from a pulsation instability, which could explain the powerful 2008 eruption, when the object brightened by a factor of $10^{4}$.

\subsubsection{Rotation of a single star}

The observed light curve could have been explained by a single K-type star rotating with a period of $\sim 1.4$ day and with two spots (or rather two groups of spots) on its surface in early seasons. The two spots would be replaced by one spot in 2007 . The object would thus belong to the FK Com class of rapidly rotating 


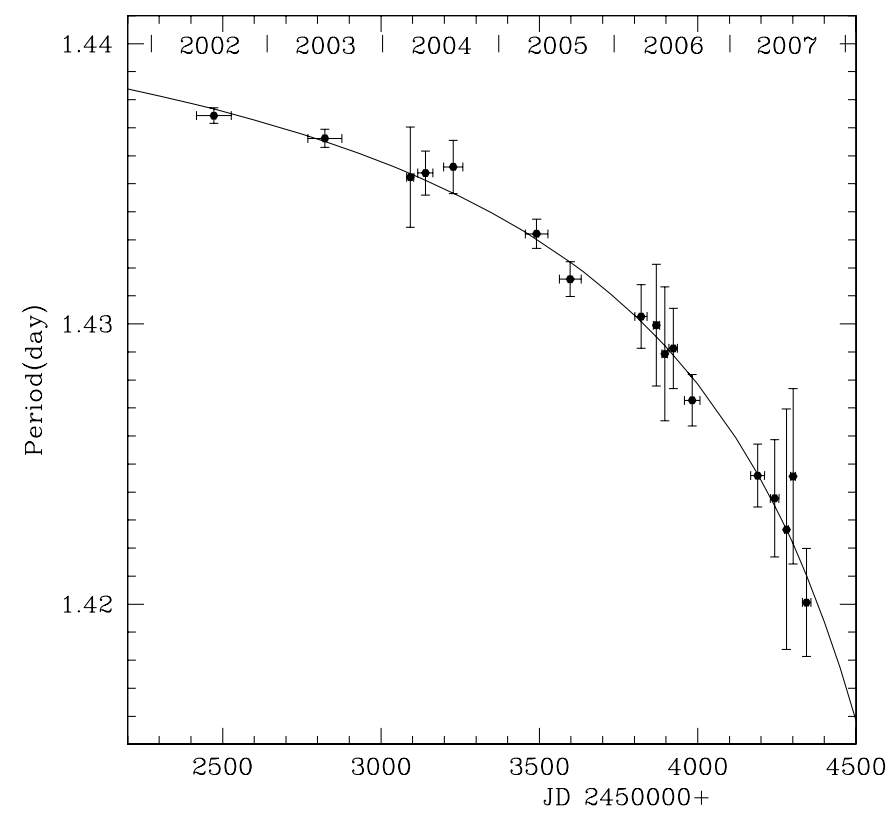

Fig. 2. Evolution of the period of the photometric variations of the V1309 Sco progenitor. The line shows a least-squares fit of an exponential formula to the data (see text and Eq. (1)).

giants (e.g. Bopp \& Stencel 1981). There are, however, several differences between the progenitor of V1309 Sco and the FK Com stars. The 1.4 day period is short, as for the FK Com stars. The very good phase stability of the observed light curve, shown in Fig. 3, implies that the spot(s) would have had to keep the same position(s) on the star surface over a time span of a few years. This seems to be very improbable given differential rotation and meridional circulation, which are expected to be substantial for a rapidly rotating star. Indeed, for the FK Com stars, the spots are observed to migrate and change their position on much shorter time scales (Korhonen et al. 2007). The systematic decrease of the rotational period is also difficult to explain for a single star, and is not observed in the FK Com stars. Finally, no eruption such as that of V1309 Sco in 2008 was observed for the FK Com stars. Indeed, there is no known mechanism that could produce such a huge eruption in a single giant, even if it were fast rotating.

\subsubsection{A contact binary evolving to its merger}

We are thus left with an eclipsing binary system. As we show below, this possibility allows us to explain all principal characteristics of the observed evolution of the V1309 Sco progenitor, as well as the 2008 outburst.

The shape of the light curve, especially in early seasons (two rounded maxima of comparable brightness and two equally spaced minima), implies that the progenitor of V1309 Sco was a contact binary. The orbital period of $\sim 1.4$ day does not allow us to classify the object as a W UMa-type binary, because the clasical W UMa stars have periods $<1$ day. Nevertheless, contact binaries with periods beyond 1 day are also observed (Paczyński et al. 2006). The exponentially decreasing period (Fig. 2) can be interpreted as resulting from an unstable phase of evolution of the system, which leads to the shrinkage of the binary orbit and finally to the merger of the system. Such a situation was
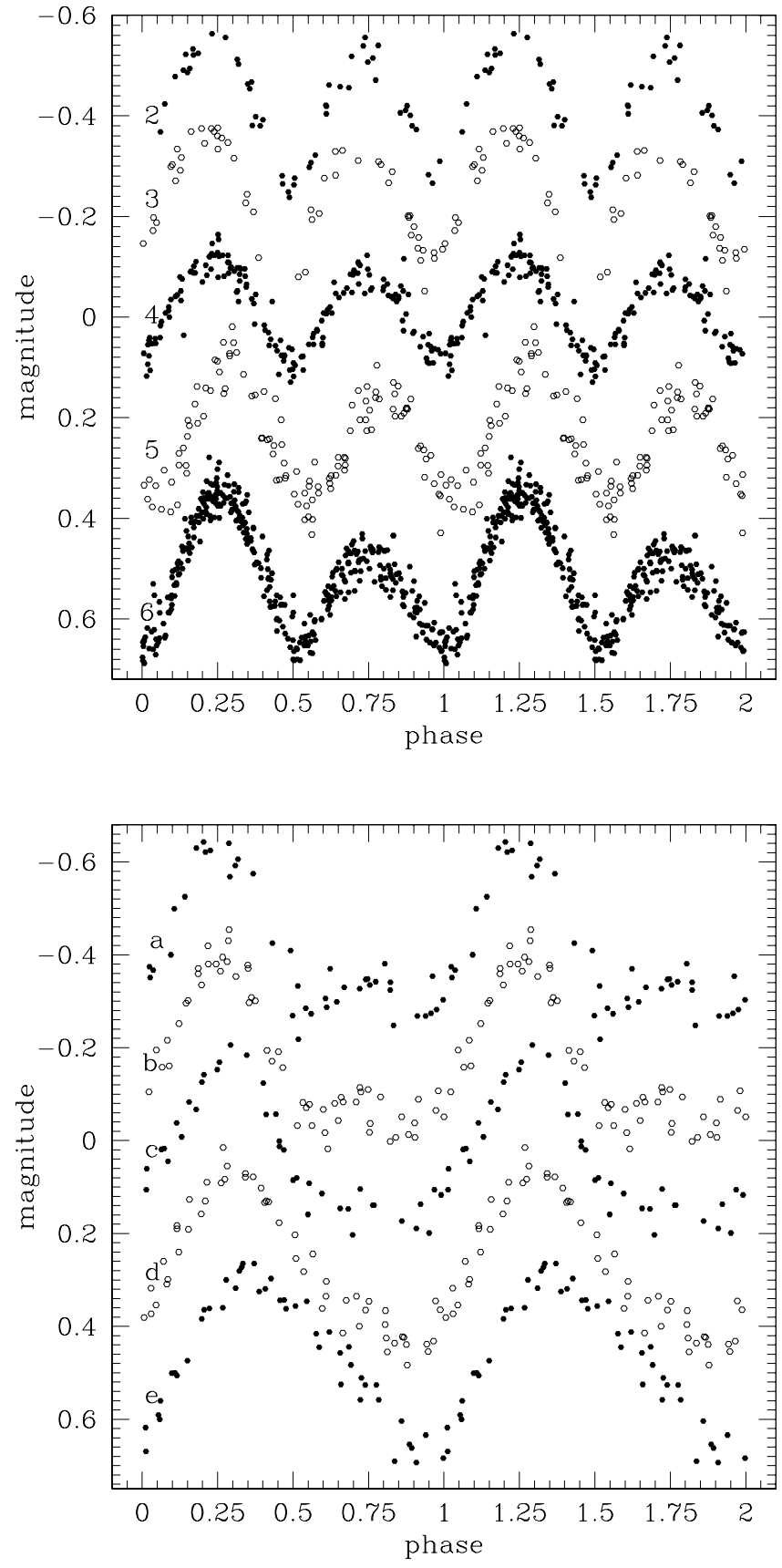

Fig. 3. Light curves obtained from folding the data with the period described by Eq. (1). Upper part: seasons 2002-2006. Lower part: season 2007 devided into five subsamples (time goes from a to e). The zero point of the magnitude (ordinate) scale is arbitrary.

predicted in theoretical studies (Webbink 1976; Robertson \& Eggleton 1977; Rasio 1995) and it is now commonly believed that the W UMa binaries end their evolution by merging into a single star. Dissipation of the orbital energy in the initial, violent phase of the merger (Soker \& Tylenda 2006) resulted in the V1309 Sco eruption observed in 2008.

One of the possible ways that can lead a binary system to merge is the so-called Darwin instability. This happens when the spin angular momentum of the system is more than a third of the orbital angular momentum. As a result, tidal interactions in the system cannot maintain the primary component in 
synchronization anymore. The orbital angular velocity is higher than the primary's angular velocity, the tidal forces increase their action and rapidly transport angular momentum from the orbital motion to the primary's rotation. For contact binaries this takes place when the binary mass ratio $q \equiv M_{2} / M_{1} \lesssim 0.1$ (Rasio 1995).

Another possibility appears to be if a binary system enters a deep contact, as discussed in Webbink (1976). This can happen, for instance, if the primary attempts to cross the Hertzsprung gap. The system then starts loosing mass and angular momentum through the outer Lagrangian point, $L_{2}$. This shrinks the binary, which further deepens the contact and increases mass and angular momentum loss. In addition the system starts orbiting faster than the components rotate. As a result, similar as for the Darwin instability, the tidal forces transport angular momentum from the orbit to the components' spins, which further accelerates the orbit shrinkage.

A maximum in the light curve of a typical contact binary is observed when we look at the system more or less perpendicularly to the line that joins the two components. As a result, we observe two maxima during each orbital revolution. The maxima are of similar brightness, because the system looks similar from both sides. The situation changes when one of the above instabilities sets in. The secondary starts orbiting faster than the primary's envelope rotates. The stars are in contact, which means that the difference in velocities is partly dissipated near the contact between the components. This should lead to the formation of a brighter (hotter) region that is visible when we look at the leading side of the secondary. The system is then brighter than when we look from the opposite direction. The maxima in the light curve start to differ. This is what we observe in the case of the V1309 Sco progenitor. As can be seen from Fig. 3, the first maximum (at phase $\sim 0.25$ ) becomes progressively stronger than the second one (phase 0.75) from 2002 to 2006. In 2007 , the second maximum disappears, and at the end of this season we observe a light curve with only one maximum and one minimum. Apparently the system evolved to a more spherical configuration with a bright (hot) spot covering a large fraction of the system's surface along the orbital plane.

\subsection{Basic parameters of the progenitor}

\subsubsection{Interstellar reddening, spectral type, and effective temperature}

The effective temperature of the progenitor can be derived from the $V-I$ colour. In 2006 a few measurements were obtained in the $V$ band. They are displayed in Fig. 4 together with the $I$ data obtained in the same time period (JD 2453 880-2453 910).

In order to determine the effective temeprature from a colour, an estimate of the interstellar reddening is also necessary. Mason et al. (2010) obtained $E_{B-V} \simeq 0.55$ from interstellar lines observed in their spectra of V1309 Sco in outburst. We constrained spectral types of the object in outburst from the UVES spectra of Mason et al. (2010) obtained in their epochs 1-3 (downloaded from the VLT archive). Comparing them with the BVRI photometry of the object obtained by the AAVSO team near the above spectral observations, we obtained $0.7 \lesssim E_{B-V} \lesssim 0.9$. The OGLE data also allowed us do derive a $V-I$ colour, equal to $5.27,223$ days after the outburst discovery date. This happened to be in the epoch of the SOAR spectroscopy of Mason et al. (2010), from which these authors derived a spectral type of M6-7. To reconcile the colour with the spectral type, $E_{B-V}>0.7$ is required. An upper limit to the extinction can be



Fig. 4. $V$ (open points) and $I$ (asterisks) measurments obtained in JD 2453 880-2 453910 (season 2006) and folded with the period described by Eq. (1). The zero point of the magnitude (ordinate) scale is arbitrary.

obtained from the Galactic Dust Extinction Service ${ }^{3}$, which estimates $E_{B-V}$ from the $100 \mu \mathrm{m}$ dust emission mapped by IRAS and COBE/DIRBE. For the position of V1309 Sco, we obtain $E_{B-V} \lesssim 1.26 \pm 0.11$. Below we assume that V1309 Sco is reddened with $E_{B-V} \simeq 0.8$.

As can be seen from Fig. 4, two $V$ measurements were taken close to the first maximum in the light curve (phase $\sim 0.26$ ), two others near the following minimum (phase $\sim 0.55$ ). The resulting colour, $V-I$, was 2.09 and 2.21 for the two phases, respectively. The mean value of the observed colour, i.e. $V-I \simeq 2.15$, corrected for the reddening and compared with the standard colours of giants (luminosity class III) results in a spectral class K1-2 and an effective temperature $T_{\text {eff }} \simeq 4500 \mathrm{~K}$ for the progenitor of V1309 Sco.

The difference in the colour, as mentiond above, implies that at the maximum the object was on average $\sim 200 \mathrm{~K}$ hotter than in the minimum. This agrees with the interpretation of the evolution of the light curve given in Sect. 5.2.3.

\subsubsection{Luminosity and distance}

The hypothesis that the progenitor of V1309 Sco was a contact binary allows us to evaluate other parameters of the system. Adopting a total mass of the binary, $\left(M_{1}+M_{2}\right)$, of 1.0-3.0 $M_{\odot}$ (observed range of masses of W UMa binaries) and an orbital period of 1.43 day, one obtains from the third Kepler law that the separation of the components, $A$, is $(3.7-5.4) \times 10^{11} \mathrm{~cm}$ $\left(5.4-7.7 R_{\odot}\right)$.

Taking the mass ratio, $q$, within the range observed in the W UMa systems, i.e. $0.07-1.0$, we can derive the radii of the Roche lobes of the components (Eggleton 1983). Assuming that both components fill their Roche lobes a total effective surface of the system can be obtained. This, along with the

${ }^{3}$ http://irsa.ipac.caltech.edu/applications/DUST/ 
above estimate of $T_{\text {eff }}$ results in a luminosity of the system of $(1.15-3.3) \times 10^{34} \mathrm{erg} \mathrm{s}^{-1}\left(3.0-8.6 L_{\odot}\right)$.

Taking into account bolometric correction, interstellar reddening, and the mean observed brightness of the V1309 Sco progenitor in 2006, $V=18.22$, we derive a distance to the object of $3.0 \pm 0.7 \mathrm{kpc}$. If the outer Roche lobes (dimensions can be calculated from Yakut \& Eggleton 2005) are assumed to be filled by the components, the luminosity increases by $\sim 35 \%$, while the distance becomes $3.5 \pm 0.7 \mathrm{kpc}$. The distance estimate is almost independent of the reddening value.

The above estimates of the luminosity and effective temperature are consistent with a $\sim 1.0 M_{\odot}$ star at the beginning of the red giant branch when they are compared with theoretical tracks of stellar evolution (e.g. Girardi et al. 2000),

\section{The outburst}

\subsection{The data}

Figure 5 (upper part) displays the rise of V1309 Sco during its eruption in 2008 (open points near maximum show the $I$ results taken from AAVSO ${ }^{4}$ ). This phase started in March 2008, and it took the object about six months to reach the maximum brightness. The rise was remarkably smooth for an eruption that led the object to brighten by a factor of $\sim 10^{4}$. We found that the light curve can be fitted by an exponential formula, i.e.

$I=-2.5 \log \left(F_{I}\right), \quad F_{I}=F_{0}+F_{1} \exp (t / \tau(t))$,

where $F_{0}=1.6 \times 10^{-7}, F_{1}=2.1 \times 10^{-8}$ are fluxes in the Vega units, $t=\mathrm{JD}-2454550$, and $\tau(t)$ is a time scale, allowed to evolve with time. The time evolution of $\tau$, resulting in the fit shown with the full line in the upper part of Fig. 5, is displayed in the lower part of the figure. These results show that during the initial $\sim 5$ months of the rise, i.e. up to JD $\simeq 2454690$, the time scale was constant and equal to $\sim 27$ days. During this phase the object brightened by $\sim 3.5 \mathrm{mag}$. Then the time scale quickly decreased and the object brightened by $\sim 6.5$ mag during a month.

The $I$ light curve of V1309 Sco during the decline observed by OGLE-IV in 2010 is presented in Fig. 6. Evidently the decline was relatively smooth at the begining of the presented phase. A similar behaviour (smooth evolution) was also observed in 2009 (see Fig. 1). However, as the decline continues in 2010, oscillations of a few hundredths magnitude on a time scale of hours appear. A periodogram analysis shows that there is no significant periodicity in these light variations.

In a few cases multiband photometric observations are available during the outburst and decline. They allow us to estimate the spectral type and effective temperature of the object. This is the case for seven dates in September 2008, when multiband photometry was made by the AAVSO team. On 4 and 16 October 2008 the object was observed by Rudy et al. (2008b) using the Infrared Telescope Facility, and JHK magnitudes were derived. These results can be combined with $B V$ AAVSO measurements and an I OGLE magnitude obtained in the same time period. On 13 April 2009, as well as in a few cases in March-April 2010 and October 2010, $V$ photometric measurements (together with $I$ ) were obtained by OGLE. On 28 August 2010 the object was observed by one of us (M.H.) with the SAAO $1.0 \mathrm{~m}$ Elizabeth Telescope with the Bessell $B V R I$ filters. The observations were reduced using standard procedures in the IRAF package. The photometry of the star was obtained with the DAOPHOT package implemented in IRAF. The

\footnotetext{
4 Available at http://www . aavso.org/
}
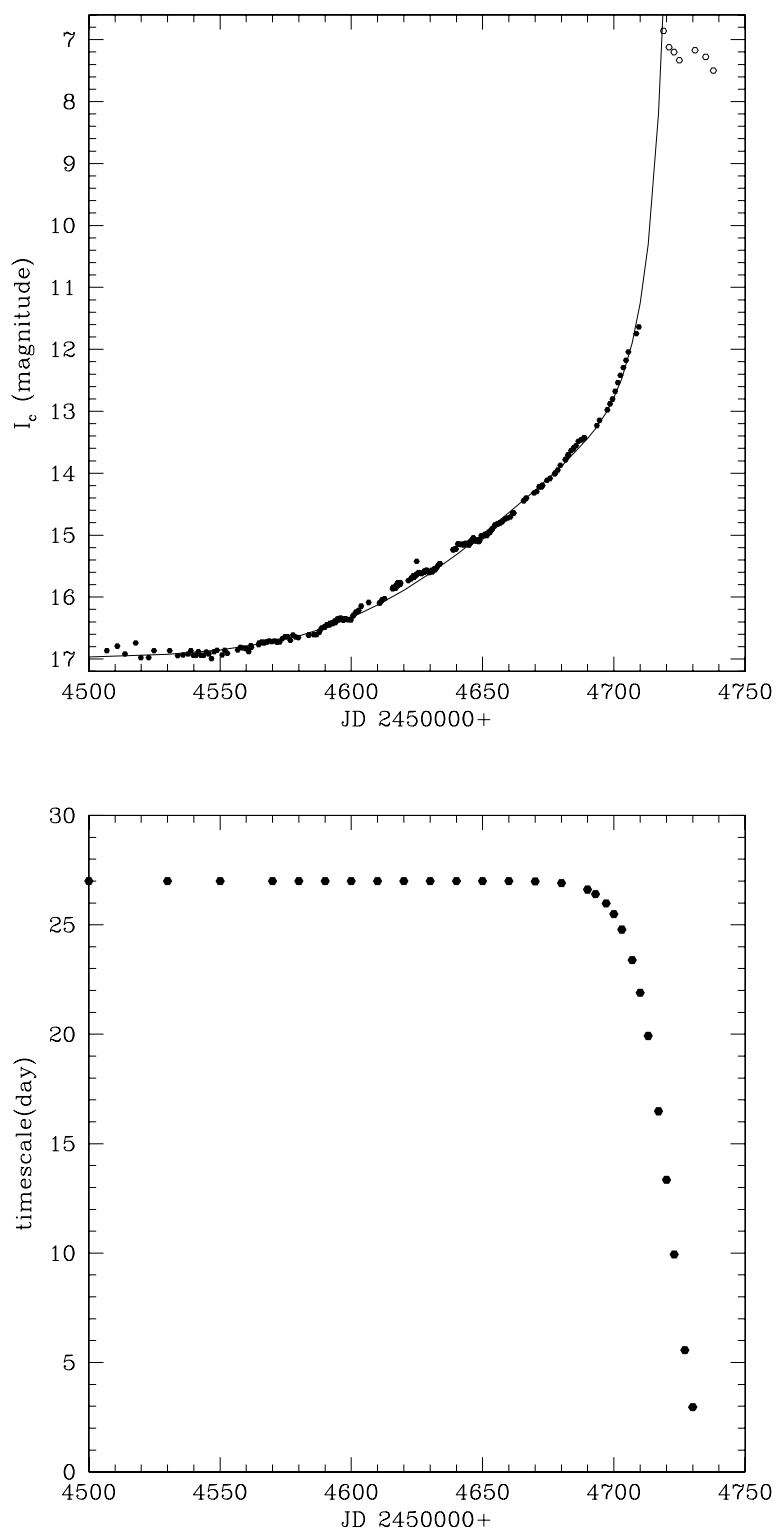

Fig. 5. I light curve of V1309 Sco during its rise to maximum in 2008 (upper part). Full points: data from OGLE III, open points: data from AAVSO. The line shows a fit of an exponential formula (Eq. (2)). The time scale used in the formula is plotted in the lower part of the figure.

resulting magnitudes are $B=22.21 \pm 0.10, V=20.42 \pm 0.13$, $R=18.84 \pm 0.10$, and $I=16.67 \pm 0.10$. The latter value agrees very well with an OGLE result $(I=16.69 \pm 0.01)$ obtained on the same date.

\subsection{Observed evolution}

Taking our estimates of the distance $(3.0 \mathrm{kpc}$, see Sect. 5.3.2) and reddening $\left(E_{B-V}=0.8\right.$, see Sect. 5.3.1), we can derive basic parameters of V1309 Sco during the outburst from multiband photometry with the same method as in Tylenda (2005). The resulting values of the effective temperature, radius, and luminosity are presented in Table 1 and Fig. 7. They show that the evolution of V1309 Sco during outburst and decline was really of the same sort as those of V838 Mon (Tylenda 2005) and V4332 Sgr (Tylenda et al. 2005). In all these cases the main 


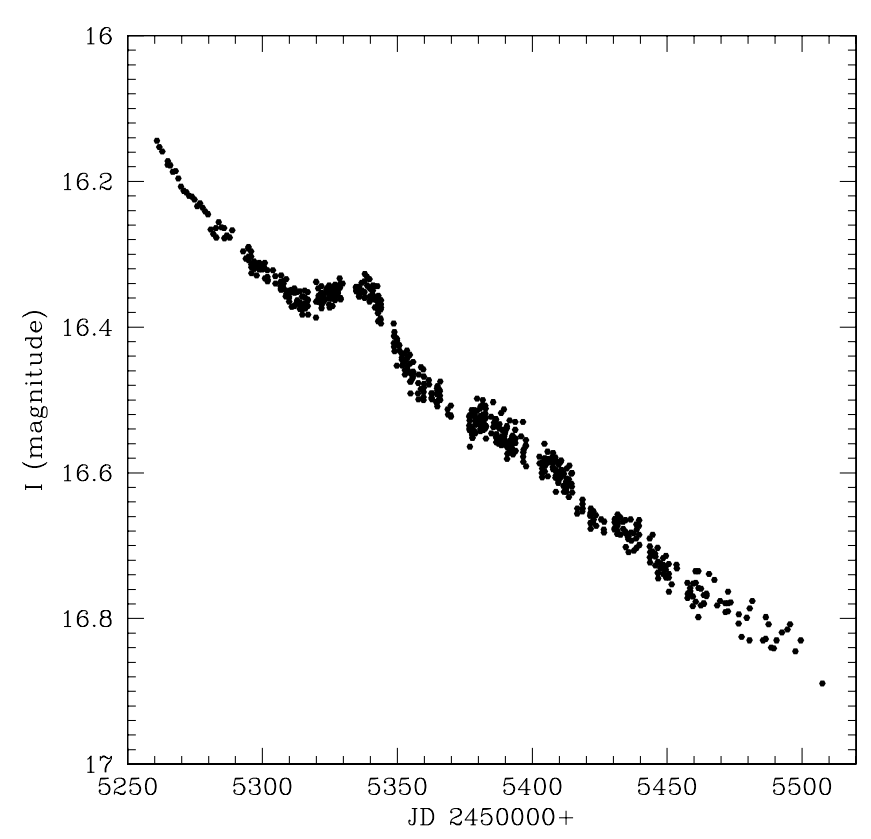

Fig. 6. $I$ light curve of V1309 Sco during the decline in 2010.

Table 1. Basic parametres of V1309 Sco in outburst and decline.

\begin{tabular}{lccccc}
\hline \hline JD & Data source & Sp.type & $T_{\text {eff }}$ & $R_{\text {eff }}\left(R_{\odot}\right)$ & $L\left(L_{\odot}\right)$ \\
\hline 4718.9 & AAVSO & F9 & 5830 & 174. & $3.16 \times 10^{4}$ \\
4721.0 & AAVSO & G1 & 5410 & 177. & $2.40 \times 10^{4}$ \\
4722.9 & AAVSO & G5 & 4870 & 209. & $2.22 \times 10^{4}$ \\
4724.9 & AAVSO & K1 & 4360 & 252. & $2.06 \times 10^{4}$ \\
4730.9 & AAVSO & K3 & 4150 & 297. & $2.36 \times 10^{4}$ \\
4735.0 & AAVSO & K4 & 3980 & 312. & $2.21 \times 10^{4}$ \\
4737.9 & AAVSO & K5 & 3900 & 297. & $1.84 \times 10^{4}$ \\
4750. & AAVSO/ & M4 & 3510 & 155. & $3.27 \times 10^{3}$ \\
& OGLE/ITF & & & & \\
4934.8 & OGLE & M7 & 3130 & 34. & 98.5 \\
5282.0 & OGLE & M5 & 3370 & 9.5 & 10.4 \\
5437.0 & SAAO & M4 & 3420 & 6.9 & 5.9 \\
5474.5 & OGLE & M3 & 3565 & 5.4 & 4.2 \\
\hline \multicolumn{7}{c}{}
\end{tabular}

decline in luminosity was accompanied by a decline in the effective temperature. In later phases of the decline the objects resume a slow increase in the effective temperature. There is no doubt that V1309 Sco was not a classical nova. We know from the present study that its eruption resulted from a merger of a contact binary. Below we show that the energy budget of the eruption can also be well accounted for by dissipation of the orbital energy of the progenitor.

\subsection{Merger-powered outburst - mergerburst}

The orbital energy and angular momentum of a binary system can be calculated from

$E_{\text {orb }}=-\frac{G M_{1} M_{2}}{2 A}=-\frac{G\left(M_{1}+M_{2}\right)^{2}}{2 A} \frac{q}{(1+q)^{2}}$

and

$J_{\mathrm{orb}}=\left(\frac{G A}{M_{1}+M_{2}}\right)^{1 / 2} M_{1} M_{2}=\left(G\left(M_{1}+M_{2}\right)^{3} A\right)^{1 / 2} \frac{q}{(1+q)^{2}}$.
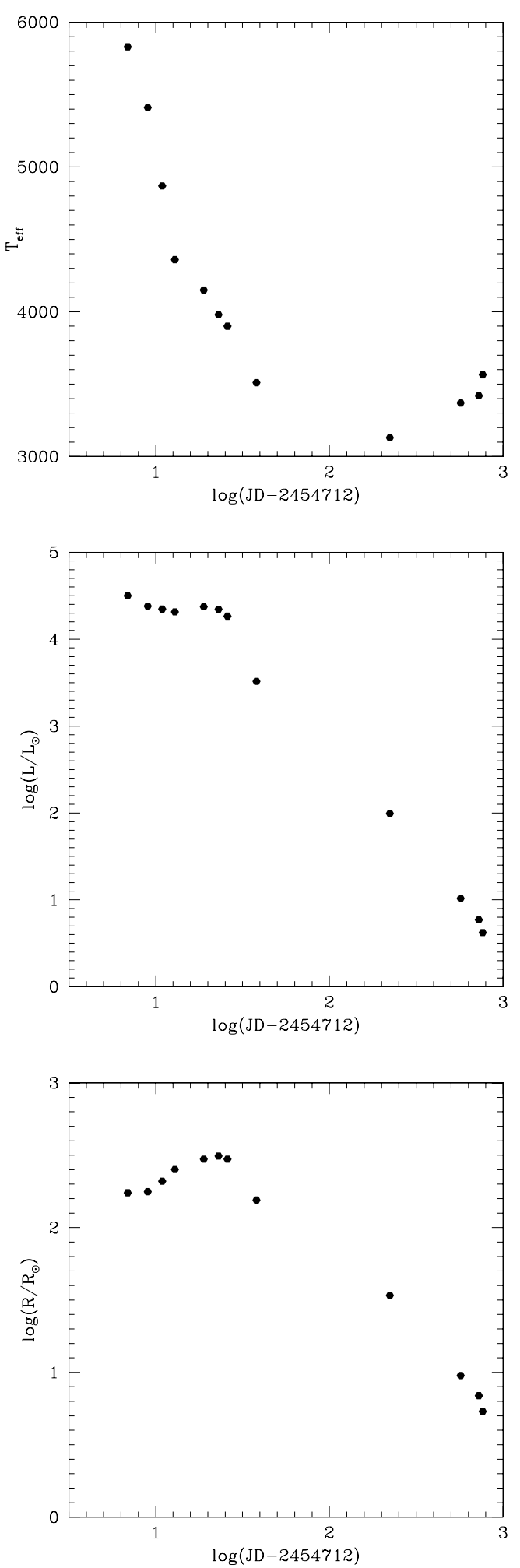

Fig. 7. Evolution of V1309 during outburst and decline (see Table 1). The abscissa displays a logarithm of time in days counted from the date of the discovery.

Taking the values assumed and obtained in Sect. 5.3.2 for the V1309 Sco progenitor, one obtains $E_{\text {orb }}=(0.2-5.6) \times 10^{47} \mathrm{erg}$ and $J_{\text {orb }}=(0.9-22.) \times 10^{51} \mathrm{~g} \mathrm{~cm}^{2} \mathrm{~s}^{-1}$.

As derived in Sect. 5.1, the orbital period decreased by $1.2 \%$ during $\sim 6$ years. Thus the system contracted by $0.8 \%$ and its 
orbital energy decreased by $\sim 10^{45}$ erg during this time period, which gives a mean rate of energy dissipation of $\sim 10^{37} \mathrm{erg} \mathrm{s}^{-1}$. Already a small portion from this can account for the observed brightening of the object between 2002 and the beginning of the 2007 season (see Fig. 1).

During the same time period the system also lost $0.4 \%$ of its orbital angular momentum. If the system started shrinking because of the Darwin instability, the orbital angular momentum loss was primarily absorbed by the spinning-up primary. If, however, the system entered a deep contact resulting from evolutionary changes of the components, mass loss through the $L_{2}$ point would be the main source of the orbital angular momentum loss. Assuming that the outflowing mass carries out a specific angular momentum of $\Omega r_{L_{2}}^{2}$, where $\Omega$ is the angular rotational velocity of the system and $r_{L_{2}}$ is a distance of $L_{2}$ from the mass centre, we can estimate that the system lost $(0.16-2.0) \times 10^{-3} M_{\odot}$ in 2002-2007. This is very much an upper limit for the mass loss. First, judging from the $\mathrm{K}$ spectral type, the system might have had magnetically active components. Then the outflowing matter might have kept corotation to radii larger than $r_{L_{2}}$ and thus have carried out a specific angular momentum larger than assumed in the above estimate. Second, matter already lost through $L_{2}$ may have formed an excretion disc around the system. Tidal interactions between the binary system and the disc can transfer angular momentum directly from the system to the disc. Third, a part of the orbital angular momentum probably went to accelerating the spins of the components.

An excretion disc is expected to be formed even if the orbital shrinkage was initiated by the Darwin instability. Decreasing separation between the components results in a deeper contact, and mass loss through $L_{2}$ must finally occur.

Apparently we see in the light curve of the V1309 Sco progenitor a signature of an excretion disc formation. We observed the system near the equatorial plane (eclipsing binary). Therefore, when the disc became sufficiently massive and thick, it could result in dimming the object for the observer. This is a likely explanation of the fading of the V1309 Sco progenitor observed during a year before the beginning of the 2008 eruption (see Fig. 1).

The progressive shortening of the orbital period finally led to engulfing the secondary by the primary's envelope. This probably took place at some point in February 2008, when the signs of the binary motion disappeared from the light curve. The secondary, or rather its core, now spiralling in the common envelope, started to release the orbital energy and angular momentum at an increasing rate. The result was the gradual and relatively gentle brightening of the object, doubling the brightness every $\sim 19$ days (Fig. 5). This phase lasted until $\sim 20$ August (JD $\simeq$ 2454700 ), when the eruption abruptly accelarated and the object brightened by a factor of $\sim 300$ during $\sim 10$ days. Perhaps this was a signature of a final disruption of the secondary's core deeply in the envelope.

In the merger process, especially during its initial, relatively gentle phases until about August 2008, mass loss probably occured mainly in directions close to the orbital plane of the progenitor. As a result, it is likely that an extended disc-like envelope was then formed, where a significant portion of the angular momentum of the progenitor was probably stored. The main eruption in August 2008, partly blocked by the envelope, was then likely to occur mainly along the orbital axis. Mason et al. (2010) interpret the line profiles observed during the outburst, especially those of the Balmer series, as produced in a partially collimated outflow and a slowly expanding shell that is denser in the equatorial plane.
As can be seen from Table 1, V1309 Sco attained a maximum luminosity of $\sim 3 \times 10^{4} L_{\odot}$. The OGLE data show that the period when V1309 Sco was brighter than $I \simeq 11$ mag lasted 40 days. Both the maximum luminosity and the time scale of the outburst are close to the theoretical expectations of a solarmass star merging with a low-mass companion (Soker \& Tylenda 2006). During 30 days, when the luminosity of V1309 Sco was $\gtrsim 10^{4} L_{\odot}$, the object radiated energy of $\sim 3 \times 10^{44} \mathrm{erg}$. This is $\$ 1 \%$ of the energy available in the binary progenitor (see above). Of course a considerable energy was also lost during the sixmonths rise, as well as in the decline after maximum. Certainly a significant amount was carried out in mass loss. Finally, an energy was also stored in the inflated remnant. Estimates made for V838 Mon show that the total energy involved in the outburst can be a factor of 10-20 higher than the energy observed in radiation (Tylenda \& Soker 2006). Even this case can be accounted for by the available orbital energy.

Our SAAO photometry performed in August 2010, as well as the OGLE $V$ and $I$ measurements obtained in 2010 (see Sect. 6.1), i.e. about two years after the outburst maximum, show that V1309 Sco reached a luminosity (see Table 1) comparable with its preoutburst value (see Sect. 5.3.2). The object was then significantly cooler however than before the outburst (early M-type spectrum versus K1-2). The latter agrees with what was observed in V838 Mon and V4332 Sgr, displaying M-type spectra several years after their outbursts (Tylenda 2005; Tylenda et al. 2005). However, the drop in luminosity of V1309 Sco was unexpectedly deep. Both V838 Mon and V4332 Sgr at present (many years after outburst) remain significantly more luminous than their progenitors. Most likely the remnant of V1309 Sco contracting after the outburst partly disappeared for us behind the disc-like envelope. Moving small-scale blobs of the envelope matter, absorbing and scattering the light from the central object, could have been responsible for the short-term variability shown in Fig. 6. The situation thus looks to be similar to that of the V4332 Sgr remnant, where the central object is most likely hidden in an opaque dusty disc (Kamiński et al. 2010; Kamiński \& Tylenda 2011).

\section{Conclusions}

The principal conclusion of our study is that all observed properties of V1309 Sco, i.e. the light curve of the progenitor during six years before the 2008 eruption, as well as the outburst itself, can be consistently explained by a merger of a contact binary. This is the first case of a direct observational evidence showing that the contact binary systems indeed end their evolution by merging into single objects, as predicted in numerous theoretical studies of these systems.

Our study also provides a conclusive evidence in favour of the hypothesis that the V838 Mon-type eruptions (red novae) result from stellar mergers, as originally proposed in Soker \& Tylenda (2003) and Tylenda \& Soker (2006). In particular, long- and short-term variabilities of the progenitors, as those of V838 Mon and V4332 Sgr (Goranskij et al. 2007; Kimeswenger 2007), which were sometimes raised as evidence against the merger hypothesis, appear now natural in view of our data for the V1309 Sco progenitor. We do not claim that all observed eruptions of the V838 Mon-type are mergers of contact binaries. There can be different ways leading to stellar mergers. What the case of V1309 Sco evidently shows is that the observational appearances of a stellar merger are indeed the same as those observed in the V838 Mon-type eruptions. 
The outburst of V1309 Sco was shorter and less luminous than those of V838 Mon and the extragalactic red novae. The latter objects attained luminosities of about or above $10^{6} L_{\odot}$ and their eruptions lasted a few months. These differences are most likely caused by the masses of merging stars. For V838 Mon, an $\sim 8 M_{\odot}$ primary was probably involved (Tylenda \& Soker 2006) instead of a $\sim 1 M_{\odot}$ one of V1309 Sco.

As noted in Sect. 5.2.3, the orbital period of the V1309 Sco progenitor of $\sim 1.4$ day is long for the observed population of contact binaries. From a study of contact binaries discovered by the OGLE project in a sky region very close to the position of V1309 Sco, Rucinski (1998) concluded that the W UMa type sequence sharply ends at the orbital period of 1.3-1.5 days (see also Paczyński et al. 2006), i.e. just at the orbital period of the V1309 Sco progenitor. This can be a pure coincidence (just one case observed), but can also indicate that binaries passing through contact at periods $\gtrsim 1$ day are not rare, but that the contact phase in their case is relatively short and quickly leads to a merger.

V1309 Sco, an overlooked object (only one research paper published so far, i.e. Mason et al. 2010), deserves much more attention of the observers and astrophysicists, as do the other V838 Mon-type objects. Apart from supernovae, they belong to the most powerful stellar cataclysms. As often happens in nature, cataclysms destroy old worlds, but also give birth to new ones. What will develop from the stellar mergers? Fast rotating giants, similar to FK Com? Peculiar stars with circumstellar discs, when new generation planets can be formed? To answer these questions, we just have to follow the evolution of the V838 Mon-type objects, V1309 Sco in particular.

Acknowledgements. The OGLE project has received funding from the European Research Council under the European Community's Seventh Framework Programme (FP7/2007-2013)/ERC grant agreement No. 246678. The research reported in this paper has partly been supported by a grant No. N N203 403939 financed by the Polish Ministery of Sciences and Higher Education. The authors are very grateful to W. Dziembowski and $\mathrm{K}$. Stẹpień for their valuable comments and suggestions when discussing various scenarios interpreting the light curve of the V1309 Sco progenitor. The periodogram analysis described in this paper was done using a ZUZA code (version 1.2/rev5) of Alex Schwarzenberg-Czerny. This paper also uses observations made at the South African Astronomical Observatory (SAAO). Thanks to the annonymous referee for the comments, which allowed us to significantly improve the quality of the paper.

\section{Appendix A: Periodograms from the preoutburst observations}

Figures A.1 and A.2 present periodograms derived from the OGLE observations of the progenitor of V1309 Sco in 2002-2007. The periodograms were obtained using the method of Schwarzenberg-Czerny (1996), which fits periodic orthogonal polynomians to the observations and evaluates the quality of the fit with an analysis of variance (AOV). The resulting AOV statistics is plotted in the ordinate of the figures.

In all seasons the dominating peaks is at a frequency of $\sim 0.7 \mathrm{day}^{-1}$, which we interpret as a frequency of the orbital period of the contact binary progenitor (Sect. 5.2). In earlier seasons, there is also a strong peak at a frequency of $\sim 1.4 \mathrm{day}^{-1}$, which is because the observations can also be reasonably fitted with quasi-sinusoidal variations having a period twice as short as the orbital period. In 2006 this peak is much weaker and practically disappears in 2007, which reflects the evolution of the light curve as displayed in Fig. 3. All other peaks are aliases resulting from combinations of the two above frequencies and a frequency of $1 \mathrm{day}^{-1}$ or subharmonics of the main peaks.
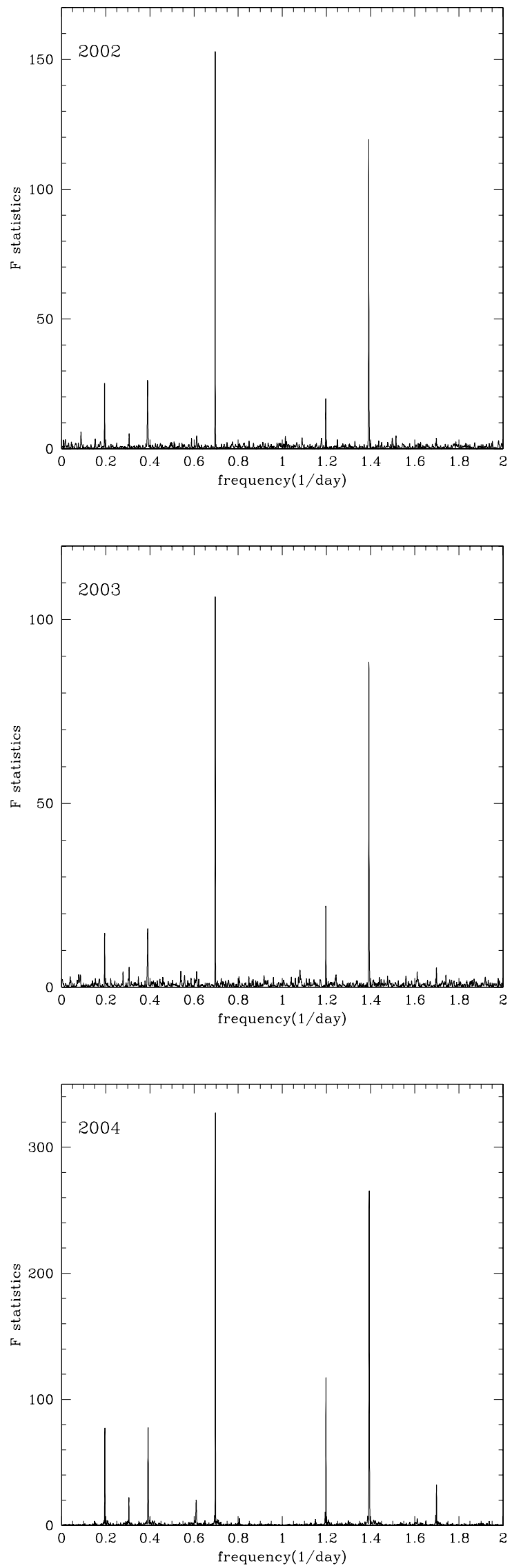

Fig. A.1. Periodograms from the observations of the progenitor of V1309 Sco in 2002-2004. 



Fig. A.2. Periodograms from the observations of the progenitor of V1309 Sco in 2005-2007.

\section{References}

Berger, E., Soderberg, A. M., Chevalier, R. A., et al. 2009, ApJ, 699, 1850

Bond, H. E., Henden, A., Levay, Z. G., et al. 2003, Nature, 422, 405

Bonnell, I. A., Matthew, R. B., \& Zinnacker, H. 1998, MNRAS, 298, 93

Bopp, B. W., \& Stencel, R. E. 1981, ApJ, 247, L131

Eggleton, P. P. 1983, ApJ, 268, 368

Girardi, L., Bressan, A., Bertelli, G., \& Chiosi, C. 2000, A\&AS, 141, 371

Goranskij, V. P., Metlova, N. V., Shugarov, S. Yu., et al. 2007, in The Nature of V838 Mon and its Light Echo, ed. R. L. M. Corradi, \& U. Munari, ASP Conf. Ser., 363, 214

Iben, I., \& Tutukov, A. V. 1992, ApJ, 389, 369

Kamiński, T., \& Tylenda, R. 2011, A\&A, 527, A75

Kamiński, T., Schmidt, M., \& Tylenda, R. 2010, A\&A, 522, A75

Kashi, A., \& Soker, N. 2011, [arXiv: 1011.1222]

Kashi, A., Frankowski, A., \& Soker, N. 2010, ApJ, 709, L11

Kimeswenger, S. 2007, in The Nature of V838 Mon and its Light Echo, ed. R. L. M. Corradi, \& U. Munari, ASP Conf. Ser., 363, 197

Korhonen, H., Berdyugina, S. V., Hackman, T., et al. 2007, A\&A, 476, 881

Kulkarni, S. R., Ofek, E. O., Rau, A., et al. 2007, Nature, 447, 458

Lawlor, T. M. 2005, MNRAS, 361, 695

Leonard, P. J. T. 1989, AJ, 98, 217

Martini, P., Wagner, R. M., Tomaney A, et al. 1999, AJ, 118, 1034

Mason, E., Diaz, M., Williams, R. E., Preston, G., \& Bensby, T. 2010 A\&A, 516, A108

Mould, J., Cohen, J., Graham, J. R., et al. 1990, ApJ, 353, L35

Munari, U., Henden, A., Kiyota, S., et al. 2002, A\&A, 389, L51

Munari, U., Henden, A., Vallenari, A., et al. 2005, A\&A, 434, 1107

Nakano, S. 2008, IAU Circ., 8972

Paczyński, B., Szczygieł, D. M., Pilecki, B., \& Pojmański, G. 2006, MNRAS, 368,1311

Rasio, F. A. 1995, ApJ, 444, L41

Robertson, J. A., \& Eggleton, P. P. 1977, MNRAS, 179, 359

Rucinski, S. M. 1998, ApJ, 115, 1135

Rudy, R. J., Lynch, D. K., Russell, R. W., et al. 2008a, IAU Circ., 8976

Rudy, R. J., Lynch, D. K., Russell, R. W., et al. 2008b, IAU Circ., 8997

Schwarzenberg-Czerny, A. 1996, ApJ, 460, L107

Shara, M. M., Yaron, O., Prialnik, D., Kovetz, A., \& Zurek, D. 2010, ApJ, 725, 831

Soker, N., \& Tylenda, R. 2003, ApJ, 582, L105

Soker, N., \& Tylenda, R. 2006, MNRAS, 373, 733

Soker, N., \& Tylenda, R. 2007, in The Nature of V838 Mon and its Light Echo, ed. R. L. M. Corradi, \& U. Munari, ASP Conf. Ser., 363, 280

Tylenda, R. 2005, A\&A, 436, 1009

Tylenda, R., \& Soker, N. 2006, A\&A, 451, 223

Tylenda, R., Crause, L. A., Górny, S. K., \& Schmidt, M. R. 2005, A\&A, 439, 651

Udalski, A. 2003, Acta Astron., 53, 291

Udalski, A., Szymański, M. K., Soszyński, I. \& Poleski, R. 2008, Acta Astron., 58,69

Webbink, R. F. 1976, ApJ, 209, 829

Yakut, K., \& Eggleton, P. P. 2005, ApJ, 629, 1055 